\title{
Temporomandibular joint arthrocentesis and lavage
}

\author{
Abstracted from \\ Guo C, Shi Z, Revington P. \\ Arthrocentesis and lavage for treating temporomandibular joint disorders. \\ Cochrane Database of Systematic Reviews 2009; Issue 4. \\ This paper is based on a Cochrane Review published in The Cochrane Library 2009, Issue 4 \\ (see www.thecochranelibrary.com for information). Cochrane Reviews are regularly updated as new evidence emerges \\ nd in response to feedback, and The Cochrane Library should be consulted for the most recent version of the review \\ Address for correspondence: Luisa Fernandez Mauleffinch, Cochrane Oral Health Group, \\ MANDEC, School of Dentistry, University of Manchester, Higher Cambridge Street, \\ Manchester M15 6FH, UK. E-mail: luisa.fernandez@manchester.ac.uk
}

\section{Question: Is arthrocentesis and lavage an effective treatment for temporomandibular joint disorders?}

Data sources The Cochrane Oral Health Group's Trials Register, Cochrane CENTRAL (Cochrane Library), Medline and Embase were used to identify likely studies. OpenSIGLE, ${ }^{*}$ CBMdisc (the Chinese Biomedical Literature Database) and the Chinese Medical Library were also searched. All the Chinese professional journals in the oral health field were searched by hand and conference proceedings were consulted. There was no language restriction.

Study selection Randomised controlled trials (RCT) and quasi-RCT that aimed to test the therapeutic effects of arthrocentesis and lavage on temporomandibular joint (TMJ) disorders were included.

Data extraction and synthesis Two review authors independently extracted data, and three review authors independently assessed the risk of bias of included trials. The first authors of the selected articles were contacted for additional information.

Results Two trials were included in the review, one of which was judged to have a high risk of bias and one to be unclear in its risk of bias. The two trials, which included 81 individuals who had TMJ disorders, compared arthrocentesis with arthroscopy. No statistically significant difference was found between the interventions in terms of pain. A statistically significant difference was found in favour of arthroscopy in maximum incisal opening (weighted mean difference of $-5.28 ; 95 \%$ confidence interval, -7.10 to -3.46 ). Mild and transient adverse reactions, such as discomfort or pain at the injection site, were reported in both groups. No data about quality of life were reported.

Conclusions There is insufficient, consistent evidence to either support or refute the use of arthrocentesis and lavage for treating patients with TMJ disorders. Further high-quality RCT of arthrocentesis need to be conducted before firm conclusions can be drawn with regard to its effectiveness.

\section{Commentary}

TMJ problems are common presentations in most oral and maxillofacial departments. If a patient fails to respond to conservative measures, surgery is not usually indicated. Arthroscopy has been

*OpenSIGLE combines the resources of major European information and document supply centres who joined in an association known as EAGLE (European Association for Grey Literature Exploitation). shown to reduce pain and improve joint movement as well as to increase inter-incisal distance. After Nitzan and co-authors eloquently described the technique of arthrocentesis in $1991,{ }^{1}$ it rapidly became popular for treating a subgroup of these patients. It has been shown in many studies that the procedure reduces the mediators of inflammation within the joint and improves the natural lubrication.

From a clinical perspective, many patients gain symptomatic relief after this simple intervention. It is surprising, but potentially explainable, why there is insufficient consistent evidence. One variable is the initial diagnosis: arthrocentesis will not be effective when people have muscle or myofascial pain, but will be effective in patients with disc displacement with or without reduction. These can be diagnosed clinically and with the benefit of magnetic resonance imaging. Other cofactors have polluted the evidence, such as the injection of steroids or sodium hyaluronate after arthrocentesis.

Many descriptive studies exist, most without controls, which show improvement after arthrocentesis. Various outcome measures have been used, but good measures do exist including pain, and inter-incisal distance and dysfunction, measured by a tool such as the Helkimo index. It would be surprising for a technique to have such widespread clinical acceptance and usage if good results were not being obtained.

In this review, only two studies could be compared, and even these were not directly comparable because of the use of adjunctive steroids in one study. A recommendation for further studies is valid, but with good consistent results from both double and single puncture arthrocentesis, it may be difficult to recruit sufficient patients.

In conclusion, arthrocentesis is a good tool in the management of the correctly diagnosed patient presenting with TMJ problems: it is simple, cheap, has little morbidity, and can be repeated or augmented with other interventions such as steroids or sodium hyaluronate.

Practice points

- The aetiology of TMJ problems is multifactorial.

- The correct diagnosis is the basis for planning treatment.

- Arthrocentesis is a low-cost low-morbidity intervention, which can be repeated.

Roger Currie

Crosshouse Hospital, Kilmarnock, Scotland, UK

1. Nitzan DW, Dolwick MF, Martinez GA. Temporomandibular joint arthrocentesis: a simplified treatment for severe, limited mouth opening. / Oral Maxillofac Surg 1991; a simplified treatment for severe, limited

Evidence-Based Dentistry (2009) 10, 110. doi:10.1038/sj.ebd.6400685 